\title{
SPAG6 silencing induces apoptosis in the myelodysplastic syndrome cell line SKM-1 via the PTEN/PI3K/AKT signaling pathway in vitro and in vivo
}

\author{
JIAXIU YIN, XINXIN LI, ZAILI ZHANG, XIAOHUA LUO, LI WANG and LIN LIU
}

Department of Hematology, The First Affiliated Hospital of Chongqing Medical University, Chongqing 400016, P.R. China

Received November 20, 2017; Accepted February 27, 2018

DOI: $10.3892 /$ ijo.2018.4390

\begin{abstract}
Apoptosis is a multi-step mechanism of cell self-destruction for maintaining cellular homeostatic balance. Accumulating evidence indicates that abnormal apoptosis promotes the evolution and progression of myelodysplastic syndromes (MDS). As a novel cancer-testis antigen, sperm-associated antigen 6 (SPAG6) has been reported to regulate apoptosis through the tumor necrosis factor-related apoptosis-inducing ligand signaling pathway in the MDS cell line SKM-1. However, the mechanism of the intrinsic cell death pathway for apoptosis induction by SPAG6 silencing is unclear. In the present study, the in vitro effects of SPAG6 silencing were investigated in SKM-1 cells through extensive biochemical and molecular approaches. Western blotting and reverse transcription-quantitative polymerase chain reaction were used to detect the expression of SPAG6 and activation of PTEN/PI3K/AKT signal pathway. Additionally, SKM-1 cells transduced with SPAG6 short hairpin RNA (shRNA) lentivirus were treated with the phosphatidylionositol 3-kinase (PI3K) inhibitor LY294002 or pan caspase inhibitor z-VAD-fmk and the apoptosis rates were measured by flow cytometry, and the expressions of associated proteins were examined by western blot analysis. A mouse xenograft model was also used to further evaluate the effects of SPAG6 knockdown on inducing tumor apoptosis in vivo. Lentivirus-mediated knockdown of SPAG6 in SKM-1 cells increased phosphatase and tensin homolog (PTEN) expression and reduced protein kinase B (AKT) phosphorylation, which in turn resulted in cell apoptosis as evidenced by induced myeloid leukaemia cell differentiation protein Mcl-1 downregulation, cytochrome $c$ release and increased caspase- 9 expression. Consistently, the PI3K inhibitor LY294002 synergistically enhanced apoptosis
\end{abstract}

Correspondence to: Dr Lin Liu, Department of Hematology, The First Affiliated Hospital of Chongqing Medical University, 1 Youyi Road, Yuzhong, Chongqing 400016, P.R. China

E-mail: liul7776@aliyun.com

Key words: myelodysplastic syndromes, sperm-associated antigen 6 gene, apoptosis, phosphoinositide 3-kinase/protein kinase B pathway, DNA methylation of SKM-1 cells when co-administered with SPAG6 shRNA lentivirus. Furthermore, treatment with the pan caspase inhibitor z-VAD-fmk failed to prevent PTEN activation upon SPAG6 knockdown, suggesting that SPAG6-regulated PTEN expression was caspase activation-independent. In addition, SPAG6 knockdown was associated with DNMT1 downregulation, implying that SPAG6 may indirectly control PTEN expression via DNA methylation. Furthermore, tumor tissues from nonobese diabetic/severe combined immunodeficient mice inoculated with SPAG6-shRNA lentivirus pre-infected SKM-1 cells exhibited significantly elevated apoptosis in the extrinsic and intrinsic pathways. These results demonstrate that SPAG6 silencing induces PTEN expression to regulate apoptosis though the PI3K/AKT pathway, indicating that SPAG6 may be a potential therapeutic target for MDS.

\section{Introduction}

Myelodysplastic syndromes (MDS) are a diverse group of disorders in which bone marrow stem and progenitor cells are damaged. In the 'pro-leukemia state', these cells are usually characterized by ineffective hematopoiesis, leading to peripheral blood cytopenia and a high risk of progression to acute myeloid leukemia (AML) in certain patients (1). Currently, the primary treatments for patients with MDS, including low-dose chemotherapy and allogeneic bone marrow hematopoietic stem cell transplantation, are unsatisfactory. MDS is associated with high mortality as it eventually progresses to AML, particularly in the cases of severe cytopenia $(2,3)$. Thus, elucidating the underlying molecular mechanisms of MDS and identifying potential targets for effective therapy is urgently required. Accumulating studies reveal that chromosomal abnormalities and abnormal gene expression have critical roles in the evolution and progression of MDS (4). In particular, patients with extrachromosomal abnormalities, such as uniparental disomy (UPD), have garnered attention from various research groups recently $(5,6)$.

UPD is the inheritance of two copies of a chromosomal region from one parent, which may generate homozygous alleles for a deleterious recessive variant. The current research has demonstrated that certain regions of UPD are only discovered in the myeloid cells of patients with MDS, and they are associated with the occurrence and development of MDS and $\operatorname{AML}(7,8)$. As one of seven genes identified in these regions 
of UPD by gene chip technology, sperm-associated antigen 6 (SPAG6) had an evidently higher expression in MDS patientderived myeloid cells than the healthy control group $(9,10)$. SPAG6 is located in the chromosomal region 10p12.2, and it was initially identified in human testis tissue with the function of regulating germ cell maturation and flagellar motility (11-13). Subsequent studies have unveiled that SPAG6 is closely associated with certain malignant diseases, including breast and lung cancer (14). Increasing evidence also indicated that, as a novel cancer-testis antigen, SPAG6 exhibits aberrant overexpression in patients with AML and displays normal levels when the patients undergo continuous remission $(9,10,15)$. Thus, SPAG6 may represent a potential therapeutic target for monitoring the evolution and progression of hematologic malignancies (16). Since the overexpression of SPAG6 has been identified in MDS patient-derived myeloid cells, we hypothesize that SPAG6 may have a significant role in the pathogenesis of MDS.

SKM-1 is an MDS cell line derived from a male patient with MDS-transformed AML (17). Our previous studies indicated that knockdown of SPAG6 by lentiviral infection significantly increased the apoptosis of SKM-1 cells by inducing the activation of specific caspases and prominent expression of tumor suppressor genes, including p53 and phosphatase and tensin homolog (PTEN) (18). Our further studies demonstrated that apoptosis initiated by SPAG6-silencing in SKM-1 cells was mediated through the death receptor pathway by upregulating the expression of Fas-associated via death domain in tumor necrosis factor-related apoptosis-inducing ligand (TRAIL) signaling (19). PTEN, as a negative regulator of the phosphatidylionositol 3-kinase (PI3K)/protein kinase B (AKT) pathway, has been demonstrated to be deleted or inactivated in various solid tumors. PTEN also has been reported to have a crucial role in regulating cellular viability though apoptosis of the mitochondrial pathway $(20,21)$, not only in solid tumors but also in MDS and AML cells (22). However, the molecular mechanisms of PTEN-induced apoptosis through the mitochondrial pathway have not been intensively defined in SKM-1 cells with SPAG6 knockdown. In the present study, whether SPAG6 silencing could induce PTEN expression and regulate apoptosis through the PI3K/AKT pathway in SKM-1 cells was determined. A mouse xenograft model was also employed to further evaluate the effects of SPAG6 knockdown on inducing tumor apoptosis in vivo.

\section{Materials and methods}

Cell culture and reagents. The MDS cell line SKM-1 was provided by Professor Jianfeng Zhou of Tongji Medical College of Huazhong University of Science and Technology (Wuhan, China). Cells were cultured in RPMI-1640 basic medium (Corning Incorporated, Corning, NY, USA) supplemented with heat-inactivated $10 \%$ fetal bovine serum (Capricorn Scientific $\mathrm{GmbH}$, Ebsdorfergrund, Germany) without antibiotic. All cells were cultured at $37^{\circ} \mathrm{C}$ in a $\mathrm{CO}_{2}$ incubator with $5 \% \mathrm{CO}_{2}$ and $95 \%$ humidified air. The culture medium was changed every 2-3 days, and cells were maintained without any significant cell morphological change or biological response. LY294002 and z-VAD-fmk, purchased from Selleck Chemicals (Houston, TX, USA), were used to treat cells $\left(5 \times 10^{4}\right.$ cells $)$ at $20 \mu \mathrm{M}$ for $1 \mathrm{~h}$.
Lentivirus production and infection. The SPAG6-targeting short hairpin RNA (shRNA) lentiviral vector (SPAG6-shRNA) and control non-specific lentiviral vector (NC-shRNA) were constructed as reported previously (23). The target sequence for SPAG6 shRNA was 5'-TGATGCTAAATTGAAGCAT-3' and that for nonsense negative control (NC) was 5'-TTCTC CGAACGTGTCACGT-3'. Lentiviral particles were produced by Shanghai GeneChem Co., Ltd. (Shanghai, China) by transiently cotransfecting lentiviral vector and helper plasmids (Addgene, Inc., Cambridge, MA, USA) into 293T cells (American Type Culture Collection, Manassas, VA, USA), followed by concentrating by ultracentrifugation for $2 \mathrm{~h}$ at $20,000 \times \mathrm{g}$ at $4^{\circ} \mathrm{C}$. Lentiviral plasmids were transfected into $293 \mathrm{~T}$ cells ( $20 \mu \mathrm{g}$ plasmids per $10 \mathrm{~cm}$ tissue culture dishes) via FuGENE ${ }^{\circledR}$ HD Transfection reagent (Promega Corporation, Madison, WI, USA), following the protocol from Addgene. SKM-1 cells at the exponential growth stage were plated in 6 -well plates $\left(5 \times 10^{4}\right.$ cells per well) and infected with SPAG6-shRNA or NC-shRNA lentivirus at a multiplicity of infection of 20 in the presence of $5 \mu \mathrm{g} / \mathrm{ml}$ polybrene. All cells were washed and resuspended in complete medium at $10 \mathrm{~h}$ after infection. The infection efficiencies and apoptosis rates of SKM-1 cells were evaluated by flow cytometry by detecting the GFP and Annexin V staining on day 5 after lentiviral infection.

Apoptosis assay by flow cytometry. Lentivirus-infected SKM-1 cells were collected, washed twice with PBS, and then resuspended in $800 \mu \mathrm{l}$ PBS. Cell apoptosis rates were detected using Annexin V and 7-aminoactinomycin D (7-AAD) double-staining, according to the manufacturer's instructions (BD Biosciences, San Jose, CA, USA). Flow cytometry was performed on a FACSCalibur machine (BD Biosciences) in the Life Science Department of Chongqing Medical University (Chongqing, China), and data were analyzed by FlowJo 7.6 software (FlowJo LLC, Ashland, OR, USA).

RNA isolation and reverse transcription-quantitative polymerase chain reaction ( $R T-q P C R)$. Total RNA was extracted with TRIzol ${ }^{\circledR}$ reagent in accordance with the manufacturer's protocol (Takara Biotechnology Co., Ltd., Dalian, China). RNA (500 ng per sample) was reverse transcribed at $42^{\circ} \mathrm{C}$ for $1 \mathrm{~h}$ into cDNA using the PrimeScript ${ }^{\mathrm{TM}} \mathrm{RT}$ reagent kit (Takara Biotechnology Co., Ltd.). PCRs were performed using a CFX-Connect Real-Time PCR system and SYBRGreen (Bio-Rad Laboratories, Inc., Hercules, CA, USA) for a total of 40 cycles, following standard assay procedures. The cycling parameters were $95^{\circ} \mathrm{C}$ for $30 \mathrm{sec}$, then 40 cycles of $95^{\circ} \mathrm{C}$ for $5 \mathrm{sec}$ and $60^{\circ} \mathrm{C}$ for $39 \mathrm{sec}$. The relative gene expression was calculated by the $2^{-\triangle \triangle \mathrm{Cq}}$ method (24), and GAPDH was used as the endogenous control gene. The PCR primer pairs were designed based on the cDNA sequences (NCBI source: Homo sapiens), and the primer sequences were as follows: SPAG6 forward, 5'-CCTTTCAGCTCTCAGTCAGGTTTC-3' and reverse, 5'-TCTTCACGTTTCATCCTTGTCCTT-3' (accession number:NM_012443.3); PTENforward,5'-ACACGACGG GAAGACAAGTT-3' and reverse, 5'-CTGGTCCTGGTATG AAGAATG-3' (accession number: NM_000314.6); DNA methyltransferase 1 (DNMT1) forward, 5'-CAGGCAGTTCA ACACCCTCATC-3' and reverse, 5'-GCTGAAGAAGCCGT 
CCCACT-3' (accession number: NM_001130823.2); GAPDH forward, 5'-CTTTGGTATCGTGGAAGGACTC-3' and reverse, 5'-GTAGA GGCAGGGATGATGTTCT-3' (accession number: NM_002046.6).

Western blot analysis. Cells were lysed on ice for $30 \mathrm{~min}$ using radioimmunoprecipitation assay lysis buffer (Beyotime Institute of Biotechnology, Haimen, China) supplemented with $1 \%$ phenylmethylsulfonyl fluoride (Beyotime Institute of Biotechnology). The protein concentrations were determined using the BCA Protein assay kit (Beyotime Institute of Biotechnology). Protein samples were denatured by boiling following mixing with loading buffer. A total of $50 \mu \mathrm{g}$ of protein per lane was separated by $8-12 \%$ sodium dodecylsulfate-polyacrylamide gel electrophoresis (Bio-Rad Laboratories, Inc.) and transferred onto polyvinylidene difluoride membranes. Following blocking for $2 \mathrm{~h}$ with $5 \%$ skim milk, or with 5\% bovine serum albumin (Biosharp, Hefei, China) for phosphorylated proteins, at room temperature, these membranes were incubated with specific antibodies overnight at $4^{\circ} \mathrm{C}$. Primary antibodies against the following proteins were used: SPAG6, caspase-8, DNMT1 (cat. nos. ab155653, ab108333, and ab188453, respectively; 1:1,000 dilution; all from Abcam, Cambridge, UK); PTEN, AKT, phospho-AKT [P-AKT(S473)], apoptosis regulator Bcl-2 (cat. nos. 9188T, 4685T, 4060T, and 3498T, respectively; 1:1,000 dilution; Cell Signaling Technology, Inc., Danvers, MA, USA); induced myeloid leukemia cell differentiation protein Mcl-1 (Mcl-1), caspase-9, cytochrome $c$ (cat. nos. sc-12756, sc-56076, and sc-13561, respectively; 1:500 dilution; Santa Cruz Biotechnology, Inc., Dallas, TX, USA); caspase-3 (cat. no. YT0656; 1:500 dilution; ImmunoWay Biotechnology Company, Plano, TX, USA); GAPDH (cat. no. AG019; 1:1,500 dilution; Beyotime Institute of Biotechnology). Subsequently, the membranes were washed with Tris-buffered saline Tween-20 (TBST) five times (5 min each) and incubated with a goat anti-rabbit or goat anti-mouse peroxidase-conjugated secondary antibody (cat. nos. A0216 and A0208, respectively; 1:3,000 dilution; Beyotime Institute of Biotechnology) for $1 \mathrm{~h}$ at room temperature. After washing with TBST another five times (5 min each), the membranes were processed to detect antigen signals with an enhanced chemiluminescence kit (Advansta, Inc., Menlo Park, CA, USA). The protein expression levels were analyzed using Vilber Fusion software (Fusion FX5 Spectra; Vilber Lourmat, Marne-la-Vallée, France).

Xenograft assays. Nonobese diabetic/severe combined immunodeficient mice (NOD/SCID; 4-5 weeks old, female, with the average weight of $17.4 \mathrm{~g}$ ) were purchased from Beijing HFK Bioscience Co., Ltd. (Beijing, China) and housed in the animal breeding facilities at the Laboratory Animal Center of Chongqing Medical University (Chongqing, China). Mice were randomly divided into two groups (5 mice per group) and were inoculated subcutaneously in the right flank with SKM-1 cells $\left(1 \times 10^{7}\right.$ cells per mouse) that were stably infected with the SPAG6-shRNA or the NC-shRNA lentivirus. A caliper was used for measuring the maximum lengths and widths of the xenografts, and the formula $0.523 \mathrm{x}$ length $\mathrm{x}$ width ${ }^{2}$ was used for calculating the tumor volumes, as reported previously (25). The mice were monitored every 3 days for signs of body weight loss and tumor growth. At 4 weeks after tumor cell inoculation, the mice were sacrificed by cervical vertebrae dislocation, and tumor tissues were collected for further analyses. All procedures involving experimental animals were approved by the University Committee on the Use and Care of Animals at Chongqing Medical University.

Immunohistochemical evaluation. Parts of each tumor tissue were fixed with $4 \%$ polyoxymethylene for $8 \mathrm{~h}$ and embedded in wax, cut into $4-\mu \mathrm{m}$-thick slices. The immunohistochemical analyses were detected using an assay kit (Beyotime Institute of Biotechnology). Following deparaffinization (three times in xylene for $15 \mathrm{~min}$, three times in ethanol for $5 \mathrm{~min}$, and three times in PBS for $3 \mathrm{~min}$ ) and permeabilization (freshly prepared $0.1 \%$ Triton X-100 in $0.1 \%$ sodium citrate for $2 \mathrm{~min}$ on ice), the sections were first put into boiled citrate solution (0.01 M, pH 6.0) for $15 \mathrm{~min}$ at $95^{\circ} \mathrm{C}$. As the temperature cooled, the sections were washed five times (3 min each) in PBS and blocked with 10\% goat serum (Beyotime Institute of Biotechnology) for $1 \mathrm{~h}$ at room temperature. Following incubation overnight with primary antibodies at $4{ }^{\circ} \mathrm{C}$, the sections were washed five times (5 min each) in PBS, and incubated with biotinylated secondary antibodies (1:1,000 dilution; cat. no. 610438, BD Biosciences) for $1 \mathrm{~h}$ at $4^{\circ} \mathrm{C}$. Primary antibodies against the following proteins were used: SPAG6, poly(ADP-ribose) polymerase (PARP; cat. nos. ab155653 and ab32138, respectively; 1:200 dilution; Abcam); cleaved-caspase-8 (Asp391; 1:200 dilution; Cell Signaling Technology, Inc.); BH3-interacting domain death agonist (BID; cat. no. YT0656; 1:50 dilution; ImmunoWay Biotechnology Company). Then, the sections were washed five times (5 min each) and then incubated with a streptavidin-peroxidase complex (ready-to-use, Zymed; Thermo Fisher Scientific, Inc., Waltham, MA, USA) for $1 \mathrm{~h}$ at room temperature. Finally, the slides were counterstained with hematoxylin (3 $\mathrm{min}$ at room temperature) and detected using diaminobenzidine (5 $\mathrm{min}$ at room temperature) under a light microscope.

Statistical analysis. All data are expressed as the mean \pm standard deviation, and the corresponding experiments were repeated with technical replicates at least three times. One-way analysis of variance was used to compare differences between multiple groups, and a least significant difference post hoc test was used for multiple comparisons for continuous variables. The Student's t-test was used to analyze differences between two groups. Statistical analysis was performed with SPSS 18.0 software (SPSS, Inc., Chicago, IL, USA). P $<0.05$ was considered to indicate a statistically significant difference.

\section{Results}

Lentivirus-mediated knockdown of SPAG6 increases PTEN expression in SKM-1 cells. Initially, experiments were performed to demonstrate that the knockdown of SPAG6 expression was achieved by lentivirus-mediated delivery of shRNA against SPAG6 in SKM-1 cells. At 5 days after viral infection, the expression of green fluorescence protein and the high infection efficiencies in both SPAG6-shRNA and control (NC-shRNA) virus-transduced SKM-1 cells demonstrated that the lentiviral particles SPAG6-shRNA and NC-shRNA 
A

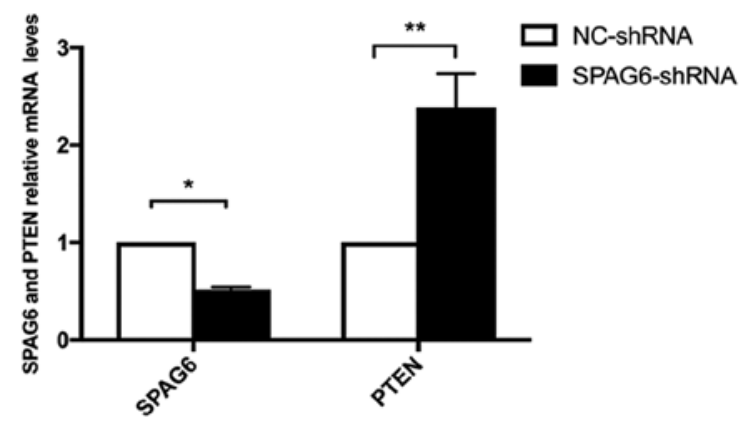

B

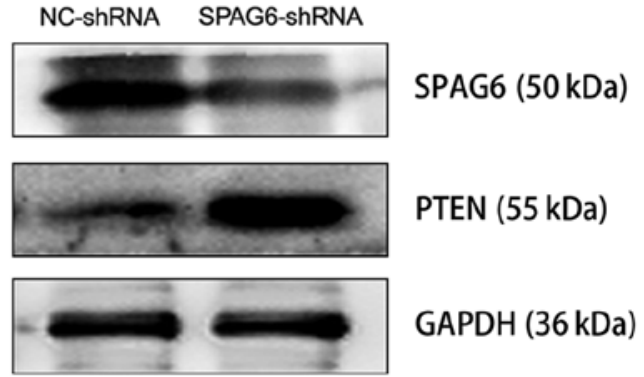

Figure 1. Lentivirus-mediated knockdown of SPAG6 increases PTEN expression in SKM-1 cells. (A) Relative mRNA levels of SPAG6 and PTEN in SKM-1 cells were determined by reverse transcription-quantitative polymerase chain reaction. The mRNA level in the control cells (NC-shRNA) was defined as 1.0 . Data are presented as the mean \pm standard deviation ( $\mathrm{n}=3$ for each group). ${ }^{*} \mathrm{P}<0.05 ;{ }^{* *} \mathrm{P}<0.01$. (B) Western blot analysis for SPAG6 and PTEN expression in SKM-1 cells at 5 days after lentiviral infection. GAPDH was used as a loading control. Data are representative of one of three independent experiments. SPAG6, sperm-associated antigen 6; PTEN, phosphatase and tensin homolog; NC, negative control; shRNA, short hairpin RNA.

A

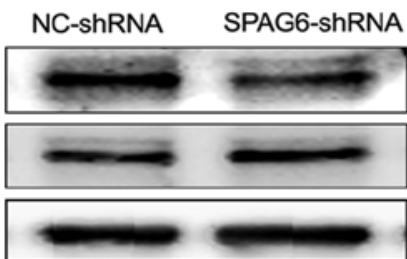

P-AKT (60 kDa)

AKT (60 kDa)

GAPDH (36 kDa)

C

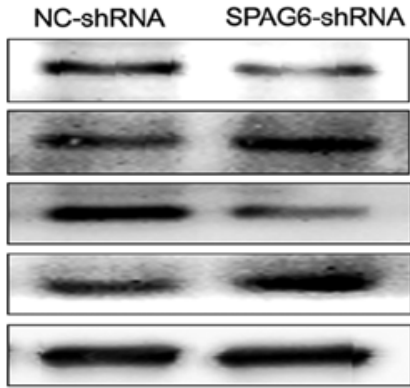

Mcl-1 (40 kDa)
B

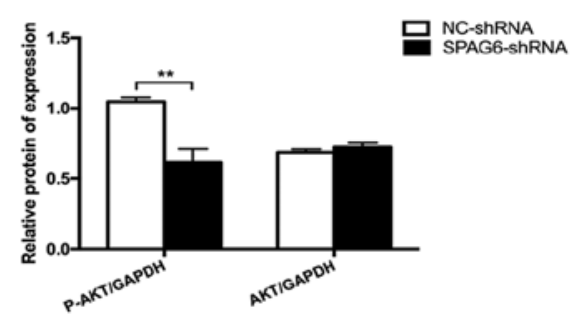

D

Mcl-1 (40 kDa)
Caspase-9 (46 kDa)
Bcl-2 (22 kDa)
Cyto C (15 kDa)
GAPDH (36 kDa)

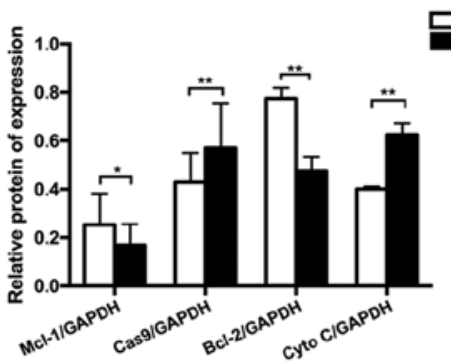

Figure 2. Knockdown of SPAG6 decreases AKT phosphorylation and promoted apoptosis in SKM-1 cells. (A) Representative western blot analysis of protein levels for total AKT and phosphorylated AKT in SKM-1 cells with and without SPAG6 knockdown. (B) Densitometry of the P-AKT and AKT western blot bands. (C) Representative western blot analysis of the Mcl-1, Bcl-2, caspase-9 and Cyto C protein levels in SKM-1 cells with and without SPAG6 knockdown. (D) Densitometry of the Mcl-1, Bcl-2, caspase-9 and Cyto C western blot bands. GAPDH was used as a loading control. Data are presented as the mean \pm standard deviation. " $\mathrm{P}<0.05 ;{ }^{* *} \mathrm{P}<0.01$. P-AKT, phospho-AKT; AKT, protein kinase B; NC, negative control; shRNA, short hairpin RNA; SPAG6, sperm-associated antigen 6; Mcl-1, induced myeloid leukemia cell differentiation protein Mcl-1; Bcl-2, apoptosis regulator Bcl-2; Cyto C, cytochrome $c$.

were able to infect SKM-1 cells effectively (data not shown), as previously reported (19). Subsequent RT-qPCR and western blot analyses also demonstrated that SKM-1 cells infected with SPAG6-shRNA virus had significantly decreased mRNA (Fig. 1A) and protein (Fig. 1B) levels of SPAG6, compared with NC-shRNA-infected cells. These results further indicate that the cell model with downregulated SPAG6 expression was successfully established. Consistent with our previous report (18), knockdown of SPAG6 substantially increased the expression of PTEN at the mRNA (Fig. 1A) and protein levels (Fig. 1B) in SKM-1 cells. These findings suggest that SPAG6 is involved in regulating PTEN expression in vitro.

Knockdown of SPAG6 decreases AKT phosphorylation and promotes apoptosis in SKM-1 cells. PTEN affects cellular apoptosis by negatively regulating the mitochondrial apoptotic pathway via PI3K/AKT signaling (26). A positive association between PTEN expression and cell apoptosis was also observed in the SKM-1 cells. As reported in a previous study (19), the SPAG6-shRNA-transduced SKM-1 cells exhibited substantially higher apoptosis rates than the NC-shRNA-transduced SKM-1 cells by the Annexin V and 7-AAD staining assays. To determine whether SPAG6 regulates apoptosis through the PI3K/AKT pathway, the activation of AKT was detected. SKM-1 cells with significantly decreased SPAG6 expression had markedly reduced P-AKT, although the total AKT levels were not significantly different to control SKM-1 cells (Fig. 2A and B). Subsequently, the expression of factors associated with the mitochondrial apoptotic pathway was examined. As shown in Fig. $2 \mathrm{C}$ and D, the protein levels of Mcl-1 and Bcl-2 were 
A

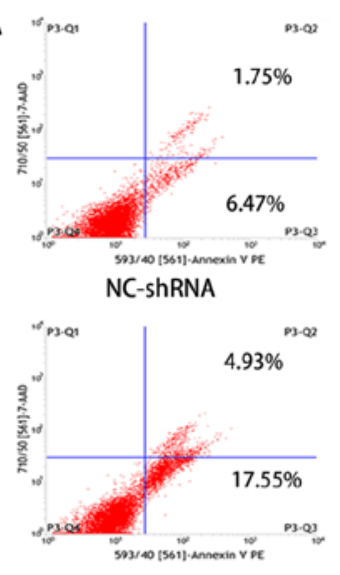

LY294002

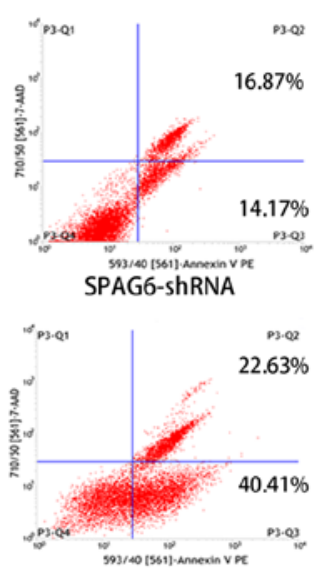

SPAG6-ShRNA+LY294002

B

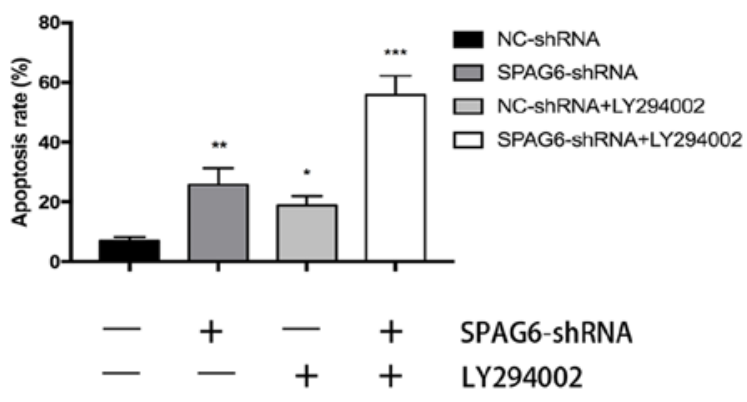

D

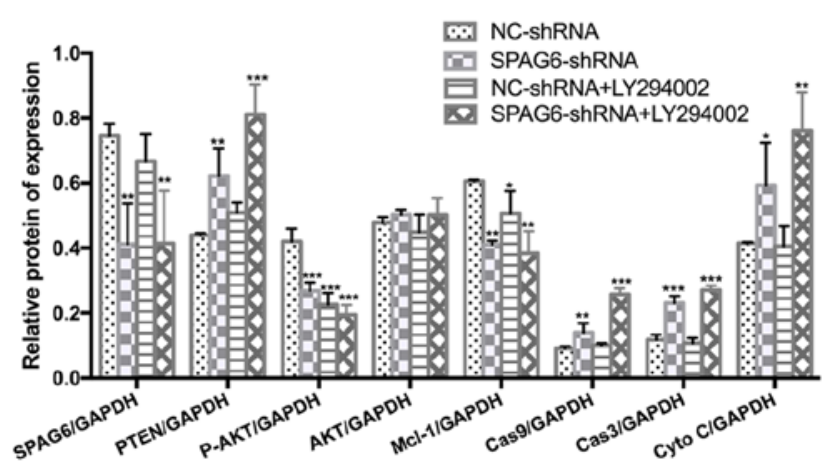

Cyto C (15 kDa)

GAPDH $(36 \mathrm{kDa})$

Figure 3. Inhibition of AKT by LY294002 enhances SPAG6 silencing-mediated apoptosis and PTEN activation. SKM-1 cells were pretreated with $20 \mu$ M LY294002 for $1 \mathrm{~h}$, followed by SPAG6-targeted (SPAG6-shRNA) or control (NC-shRNA) lentiviral infection. (A) Representative flow cytometry profile of Annexin V and 7-aminoactinomycin D staining in SKM-1 cells with different treatments. (B) Summary of the apoptosis rates in SKM-1 cells with different treatments. (C) Western blot analysis of the SPAG6, PTEN, P-AKT, AKT, MCL-1, caspase-9, caspase-3, and cytochrome $c$ protein levels in SKM-1 cells with different treatments. GAPDH was used as a loading control. (D) Summarization of densitometry of the western blot bands. ${ }^{*} \mathrm{P}<0.05$, ${ }^{* *} \mathrm{P}<0.01,{ }^{* * *} \mathrm{P}<0.001$, vs. NC-shRNA alone. All data are expressed as the mean \pm standard deviation ( $\mathrm{n}=3$ for each group). NC, negative control; shRNA, short hairpin RNA; SPAG6, sperm-associated antigen 6; PTEN, phosphatase and tensin homolog; P-AKT, phospho-AKT; AKT, protein kinase B; Mcl-1, induced myeloid leukemia cell differentiation protein Mcl-1; Cas, caspase; Cyto C, cytochrome $c$.

lower in SPAG6-shRNA cells compared with control cells, while the levels of caspase-9 and cytochrome $c$ were higher in the SPAG6-shRNA group than in the control group. Taken together, the results indicated that SPAG6 regulated apoptosis by activating the PTEN/PI3K/AKT-mediated mitochondrial apoptotic pathway.

PI3K/AKT inhibition and SPAG6 silencing synergistically promotes apoptosis in SKM-1 cells. To determine whether PTEN activation and AKT inactivation were sufficient for induction of apoptosis in SKM-1 cells, the PI3K inhibitor LY294002. As shown in Fig. 3A and B, the apoptosis rates of LY294002-treated and SPAG6-shRNA lentivirus-transduced SKM-1 cells were significantly higher than that in the control cells. Notably, cotreatment with LY294002 and SPAG6-shRNA markedly promoted apoptosis, and these SKM-1 cells displayed a higher apoptotic rate than any of the other groups. Western blot analysis indicated that the PTEN level in cells infected with SPAG6-shRNA lentivirus alone was higher than that in cells treated with LY294002 alone, although it was significantly lower than that in cells cotreated with LY294002 and SPAG6-shRNA lentivirus (Fig. 3C and D). Correspondingly, an opposing pattern of changes for AKT phosphorylation was observed, which was attributed to the negative regulation of AKT activity by PTEN (Fig. 3C and D). Furthermore, co-administration of LY294002 significantly enhanced SPAG6shRNA lentivirus-mediated Mcl-1 downregulation, caspase-9 and caspase-3 activation, and cytochrome $c$ release in SKM-1 cells (Fig. 3C and D). Taken together, these findings suggest that PTEN-mediated AKT inactivation has a critical role in enhancing apoptosis in SKM-1 cells with SPAG6 knockdown.

SPAG6 knockdown-induced PTEN upregulation is independent of caspase activation. Apoptosis can be initiated 
A

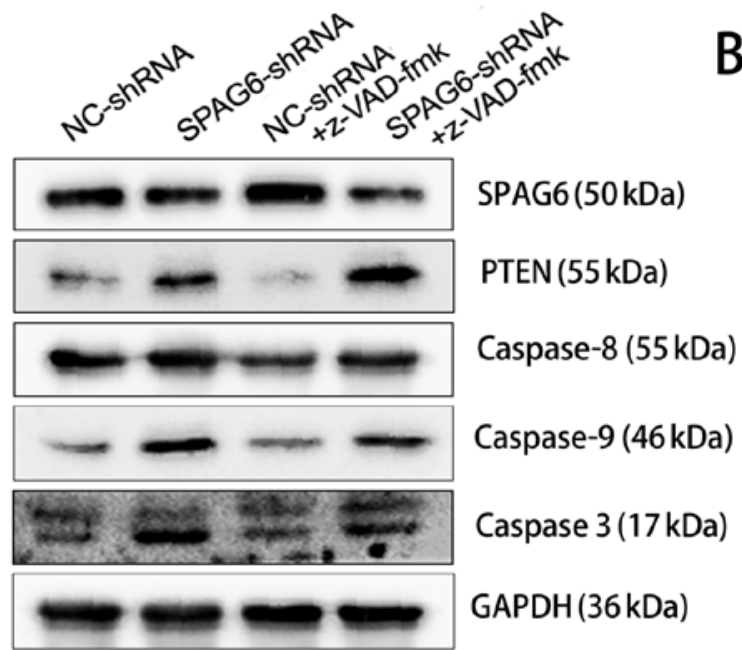

B

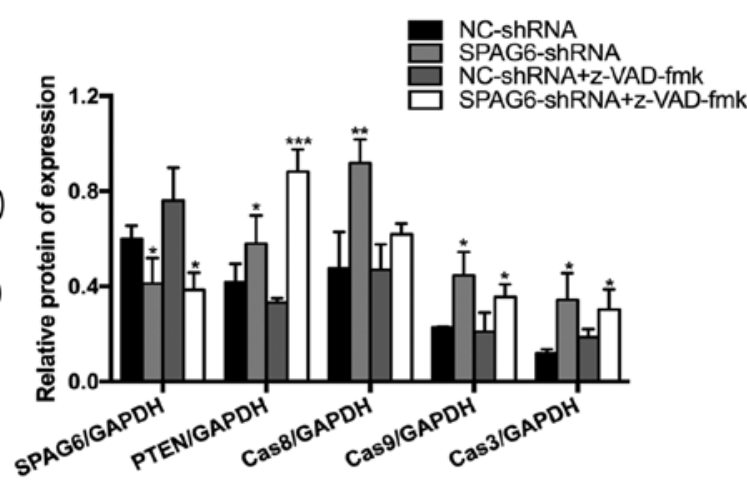

C

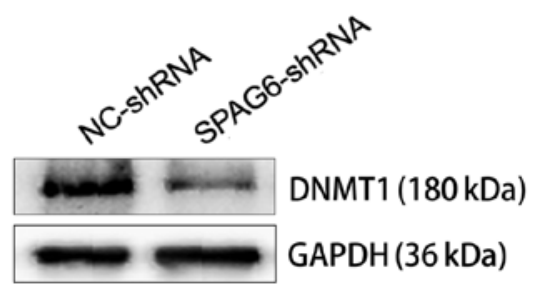

D

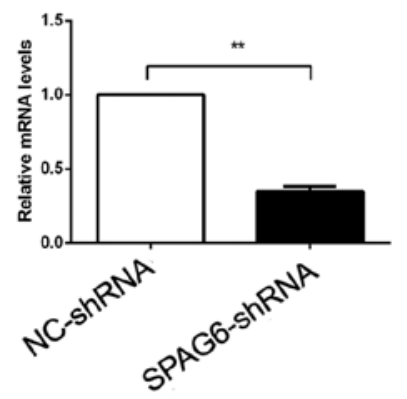

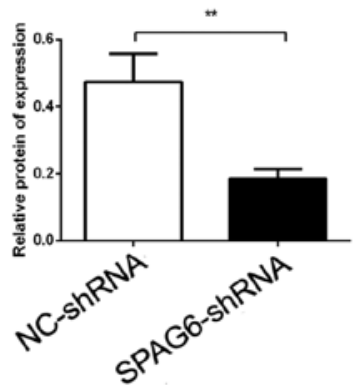

Figure 4. SPAG6 knockdown-induced PTEN upregulation is caspase activation-independent and associated with DNMT1 downregulation. SKM-1 cells were pretreated with z-VAD-fmk $(20 \mu \mathrm{M})$ for $1 \mathrm{~h}$, followed by SPAG6-targeted (SPAG6-shRNA) or control (NC-shRNA) lentiviral infection. (A) The protein levels of SPAG6, PTEN, caspase-8, caspase-9, and caspase-3 in SKM-1 cells with different treatments were determined by western blot analysis. GAPDH was used as a loading control. (B) Summarization of densitometry of the western blot bands. (C) Western blot analysis for DNMT1 expression in SKM-1 cells with or without SPAG6 knockdown. GAPDH was used as a loading control. (D) Relative expression of DNMT1 at both the mRNA (left) and protein (right) levels. All data are presented as the mean \pm standard ( $\mathrm{n}=3$ for each group). ${ }^{*} \mathrm{P}<0.05,{ }^{* * *} \mathrm{P}<0.01,{ }^{* * *} \mathrm{P}<0.001$, vs. NC-shRNA alone. NC, negative control; shRNA, short hairpin RNA; SPAG6, sperm-associated antigen 6; PTEN, phosphatase and tensin homolog; Cas, caspase; DNMT1, DNA methyltransferase 1.

through either death receptor activation or the mitochondrial apoptotic pathway. The cleavage/activation of caspase- 8 and PARP also downregulate AKT activity and indirectly activate the mitochondrial apoptotic pathway (27). Thus, it is possible that PTEN activation followed by dephosphorylation of AKT in SKM-1 cells with SPAG6 knockdown is caused by caspase activation. To elucidate the role of caspase activation in PTEN activation, SKM-1 cells were treated with z-VAD-fmk, a cell-permeant pan-caspase inhibitor, to block the caspase-dependent apoptotic pathway prior to knockdown of SPAG6. Western blot analysis demonstrated that although z-VAD-fmk slightly inhibited the expression of total caspase-8/9, it did not block PTEN activation in SKM-1 cells with silenced SPAG6 (Fig. 4A and B). In the presence of z-VAD-fmk, knockdown of SPAG6 was still able to increase the level of total caspase-8/9/3 compared with z-VAD-fmk + NC-shRNA treatment, suggesting the progression of cell apoptosis. These data clearly indicate that caspase activation is a consequence of SPAG6 silencing-mediated AKT inactivation, but not the cause of PTEN activation in SKM-1 cells.

PTEN activation in SKM-1 cells with SPAG6 knockdown is associated with reduced expression of DNMT1. There is growing evidence indicating that DNA methylation can silence tumor suppressors through high expression of DNA methyltransferases (DNMTs) to facilitate tumorigenesis and tumor development (28). To explore whether DNMTs have a role in upregulating PTEN expression, the expression level of DNMT1 was determined by RT-qPCR and western blot analysis. Consistently, the mRNA and protein levels of DNMT1 were significantly decreased in SKM-1 cells infected with SPAG6-shRNA lentivirus, compared to that in the control cells (Fig. 4C and D). Thus, potentially a positive association between SPAG6 and DNMT1 expression may contribute to SPAG6 silencing-induced PTEN activation.

Knockdown of SPAG6 inhibits tumor growth and induces apoptosis in a SKM-1 xenograft mouse model. To determine whether the in vitro findings were applicable in vivo, NOD/ SCID mice were inoculated with SKM-1 cells with or without SPAG6 knockdown by subcutaneous injection. Immunohistochemical analyses and western blotting were performed to evaluate the morphological changes and induction of apoptosis in tumor cells (Fig. 5). The tumor sizes and tumor weights of the mice inoculated with SKM-1 cells pre-infected by SPAG6-shRNA lentivirus were significantly less than those of the control mice (Fig. 5A). As reported in our 
A

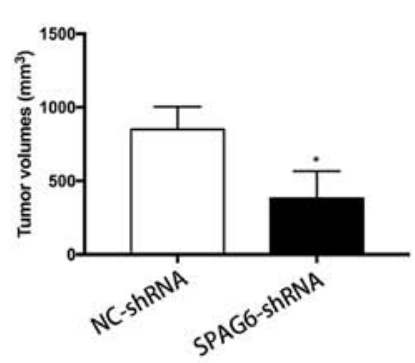

B
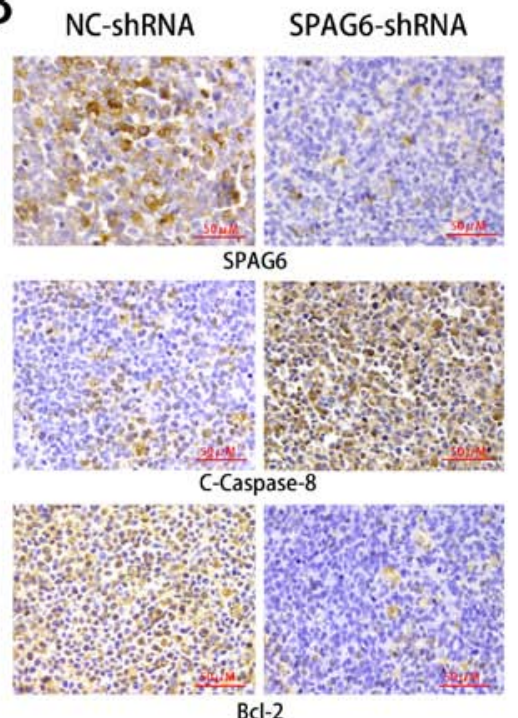

$\mathrm{BCl}-2$

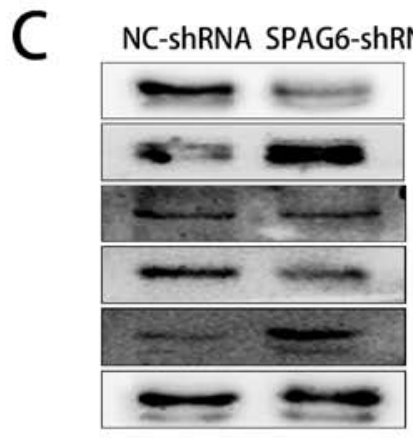

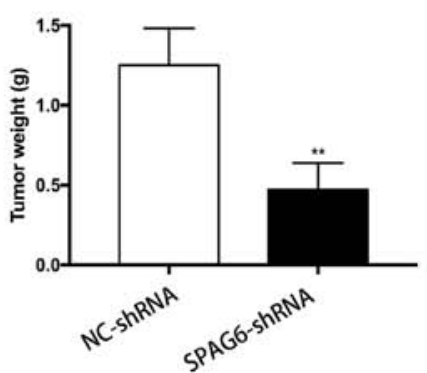

NC-shRNA

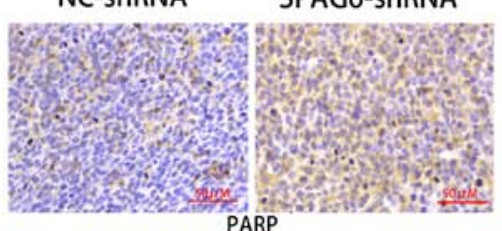

PARP

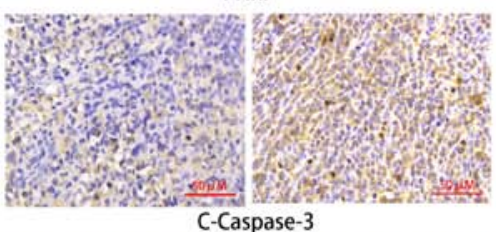

C-Caspase-3

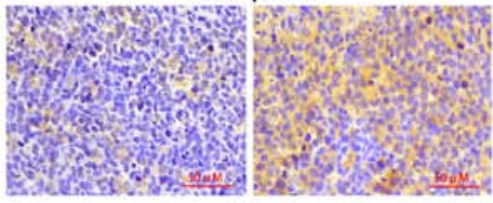

BID

D

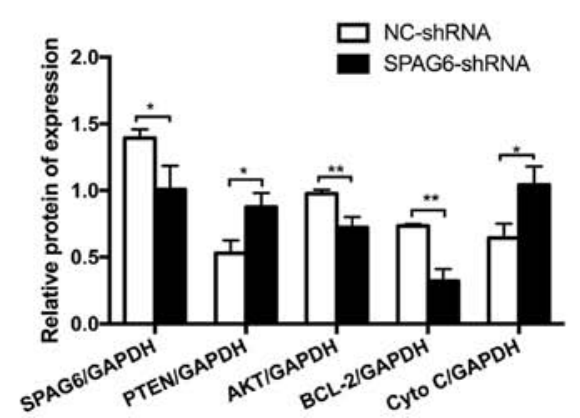

Figure 5. In a SKM-1 xenograft mouse model, silencing of SPAG6 induces PTEN/PI3K/AKT signaling-mediated apoptosis of tumor cells. (A) Comparison of the sizes and weights of tumors from the mice inoculated with lentivirus pre-infected SKM-1 cells at 4 weeks after inoculation. (B) Representative images showing the immunohistochemistry staining results for SPAG6 and apoptosis-associated molecules including PARP, Bcl-2, BID, cleaved caspase-8, and cleaved caspase-3 (x20). (C) Western blot analysis for the expression of SPAG6, PTEN, AKT, Bcl-2, and Cyto C in tumor tissues from mice inoculated with SKM-1 cells that were pre-infected with SPAG6-tageting shRNA or control shRNA lentivirus. GAPDH was used as a loading control. (D) Summarization of densitometry of the western blot bands. Data are representative results from at least three mice per group. Data are presented as the mean \pm standard deviation ( $\mathrm{n}=5$ for each group in $\mathrm{A} ; \mathrm{n}=3$ for each group in $\mathrm{D}$ ). ${ }^{*} \mathrm{P}<0.05,{ }^{* *} \mathrm{P}<0.01$, vs. NC-shRNA. NC, negative control; shRNA, short hairpin RNA; SPAG6, sperm-associated antigen 6; PARP, poly(ADP-ribose) polymerase; C-, cleaved-; BID, BH3-interacting domain death agonist; PTEN, phosphatase and tensin homolog; Cyto C, cytochrome $c$.

previous study (18), tumor sections from the mice inoculated with SPAG6-shRNA-transduced SKM-1 cells showed an apparently necrotic morphology, infiltration of inflammatory cells, disordered and irregular tumor cell arrangement, and tumor cells with an increased nuclear cytoplasmic ratio. In addition, the number of dark brown-colored apoptotic cells from the SPAG6-shRNA lentivirus group was significantly higher than that from the NC-shRNA lentivirus group (18). Furthermore, tumor cells with SPAG6 knockdown exhibited evident signs of cell apoptosis, as indicated by significantly altered signals from the results of immunohistochemical staining for apoptosis-associated molecules, including BID, PARP, Bcl-2, cleaved-caspase-8, and cleaved-caspase-3 between these two groups (Fig. 5B). The western blot results further substantiated the upregulation of pro-apoptotic factors like cleaved-caspase-8, cleaved-caspase-3, PARP and BID, and downregulation of the anti-apoptotic factor Bcl-2 in tumor cells with SPAG6 knockdown (Fig. 5C and D). Collectively, these findings support that that SPAG6 knockdown induced apoptosis of SKM-1 cells via the extrinsic and intrinsic 
pathways during in vitro culturing and in the in vivo xenograft mouse model.

\section{Discussion}

Despite a hypercellular or normal marrow, the majority of patients with early-stage MDS exhibit peripheral blood cytopenia, which has been ascribed to excessive programmed cell death of hematopoietic cells $(29,30)$. However, the progression of MDS in the late stage may be attributed to cellular overproliferation and relatively reduced apoptosis by abnormal gene expression or inactivation of certain tumor suppressor genes (31). PTEN, a well-established tumor suppressor that negatively regulates PI3K/AKT signaling, has been reported to have low expression in, and contribute to the development of, various malignancies, including prostate, ovarian, and breast cancer (32-34). The genetic abnormalities of PTEN have a crucial role in inducing the disease progression from low-risk MDS to high-risk MDS, and eventually to acute leukemia (35).

The formation and development of various malignant tumors are largely associated with uncontrolled cell proliferation and abnormal apoptosis. In general, the death receptor pathway and the mitochondrial pathway have been reported to control programmed cell death to maintain the balance of tissue homeostasis (36). Our previous study demonstrated that silencing of SPAG6 promoted cell apoptosis through the death receptor pathway by altering the TRAIL signals in the MDS cell line SKM-1 (19). On the other hand, decreased SPAG6 expression was associated with increased expression of the tumor suppressor gene PTEN in SKM-1 cells. Additionally, the expression of molecules in the PI3K/AKT pathway were detected, which function as survival signals to support tumor growth. Significant AKT dephosphorylation and evident signs of cell apoptosis (MCL-1 downregulation, caspase-9 activation, cytochrome $c$ release) were observed following SPAG6 knockdown in SKM-1 cells. Thus, in addition to the TRAIL signaling pathway, the PTEN/PI3K/AKT pathway also contributed to SPAG6 silencing-initiated apoptosis in SKM-1 cells.

AKT, a serine-threonine kinase, is activated by PI3K to interfere with cell survival via the apoptotic machinery. The activity of PI3K can be antagonized by the tumor suppressor PTEN through removal of the 3-phosphate from phosphatidylinositol 3,4,5-trisphosphate $(37,38)$. AKT regulates cell apoptosis by directly or indirectly inducing pro-apoptotic or inhibiting anti-apoptotic Bcl-2 family proteins and releasing cytochrome $c$ to initiate the mitochondrial pathway. SKM-1 cells co-administered with the PI3K inhibitor LY294002 and SPAG6-shRNA lentivirus exhibited a higher apoptosis rate than SKM-1 cells with either single treatment. As in the LY294002 group, the treatment of cells with SPAG6shRNA lentivirus reduced AKT phosphorylation, compared to the control group. The synergistic effect of LY294002 and SPAG6-shRNA on cell apoptosis was attributed to AKT inactivation, which was also supported by superimposed elevation of PTEN expression. This observation further substantiates that SPAG6 regulates PTEN activation and subsequent AKT phosphorylation to control cell survival via a mitochondrial apoptotic pathway in SKM-1 cells.
Our previous study also indicated that SPAG6 knockdown induced the activation of caspase-8, which facilitated the cleavage of BID to form truncated BID, and mediated the activation of caspase-3 and caspase-9 in SKM-1 cells (19). This raised the possibility that AKT dephosphorylation might be the indirect consequence of caspase activation-induced PTEN expression (27). To determine whether PTEN activation is crucial for cell apoptosis though the PI3K/AKT pathway, we treated SKM-1 cells with the broad caspase inhibitor z-VAD-fmk to abrogate caspase-8-mediated cleavage/activation of BID and caspase-9. However, the increased PTEN expression was not abrogated following caspase inhibition. Furthermore, the observation of increased caspase-9 protein in z-VAD-fmk-treated SPAG6 knockdown cells suggested that PTEN-mediated AKT dephosphorylation promoted apoptosis through the mitochondrial apoptotic pathway rather than the caspase-dependent pathway. Furthermore, the investigations using the xenograft mouse model reached the same conclusions as the in vitro study.

Apoptosis can be triggered in a multistep pathway by extracellular stimuli and intracellular signals. Several studies have demonstrated that PTEN has a relatively low expression level in myeloid cells of patients with MDS (39). The PI3K inhibitor LY294002 enhanced PTEN activation and the apoptotic rate in SKM-1 cells, while the caspase inhibitor z-VAD-fmk failed to prevent PTEN activation. A potential explanation is that various signaling pathways may be involved in regulating apoptosis. In addition, the expression of PTEN is induced not only by caspase-dependent cleavage/activation but also by other pathways, such as the nuclear factor- $\kappa \mathrm{B}$, c-Jun $\mathrm{N}$-terminal kinases and p53 pathways. Furthermore, growing evidence demonstrates that inactivation of tumor suppressor genes is associated with hypermethylation, rather than intragenic mutations (28).

From an epigenetics standpoint, DNA methylation may suppress the expression of PTEN by DNMTs in human MDS (40). A recent study also indicate that DNA methylation is involved in the transcriptional regulation of SPAG6 in non-small cell lung cancer (41). However, the association between SPAG6 expression and DNA methylation in MDS remains unreported. As is well established, two different processes of methylation occur: One is de novo methylation, which establishes the methylation state, and DNMT3a and DNMT3b were identified as predominantly responsible for this process in mammals; the other method is maintenance methylation, which copies the pattern onto daughter DNA strands following DNA replication, and DNMT1 was identified as predominantly responsible for this process. The first mammalian MT discovered was DNMT1, which is highly conserved among eukaryotes $(42,43)$. To the best of our knowledge, the current study if the first to report that SPAG6 silencing decreases DNMT1 expression and subsequently upregulates PTEN expression. However, additional in-depth studies are required for further elucidation of the mechanisms of SPAG6-mediated PTEN activation. Furthermore, there is an inherent limitation using a cell line to study the role of SPAG6 in cell apoptosis, as patients with MDS undergo disease progression over time. As such, the current xenograft mouse model may not accurately reflect the progression of MDS. Therefore, a murine xenotransplant model is required 
for future investigations on how SPAG6 impacts MDS disease progression.

In summary, the data suggest that SPAG6 has a critical role in the PTEN-mediated PI3K/AKT pathway to affect apoptosis in the SKM-1 human MDS cell line. Knockdown of SPAG6 also inhibits tumor growth of SKM-1 xenografts by inducing cell apoptosis via extrinsic and intrinsic pathways in vivo. Collectively, these data support that SPAG6 silencing induces PTEN activation and AKT inactivation, which in turn results in cell apoptosis in the mitochondrial pathway. Since the therapeutic efficacy of current MDS treatments is not ideal in the clinic, the findings of the current study suggest that SPAG6 might be a promising putative molecular target for advancing the treatment of MDS.

\section{Acknowledgements}

We thank Ms. Shaoqiu Jiang, Ms. Huan Yan, Ms. Hui Gou, Ms. Tingting Li and Ms. Yao Ding (The First Affiliated Hospital of Chongqing Medical University, Chongqing, China) for their assistance. We thank the service provided by the Chongqing Key Laboratory of Translational Medicine in Major Metabolic Diseases (The First Affiliated Hospital of Chongqing Medical University, Chongqing, China).

\section{Funding}

The Natural Science Foundation of Chongqing (grant no. CSTC2013jjB0145) and the National Natural Sciences Foundation of China (grant no. 81570109).

\section{Availability of data and materials}

The datasets used or analyzed during the current study are available from the corresponding author on reasonable request.

\section{Authors' contributions}

LW, LL and XLi contributed to the conception of the study. JY and LL performed the experiments data analyses and wrote the manuscript. XLu and ZZ helped perform the analysis with constructive discussions.

\section{Ethics approval and consent to participate}

All applicable international, national, and/or institutional guidelines for the care and use of animals were followed. All procedures performed in studies involving animals were in accordance with the ethical standards of the institution or practice at which the studies were conducted and approved by the University Committee on the Use and Care of Animals at Chongqing Medical University (Chongqing, China).

\section{Consent for publication}

Not applicable.

\section{Competing interests}

The authors declare that they have no competing interests.

\section{References}

1. Greenberg PL, Stone RM, Al-Kali A, Barta SK, Bejar R, Bennett JM, Carraway H, De Castro CM, Deeg HJ, DeZern AE, et al: Myelodysplastic syndromes, version 2.2017, NCCN Clinical Practice Guidelines in Oncology. J Natl Compr Canc Netw 15: 60-87, 2017.

2. Li N, Chen Q, Gu J, Li S, Zhao G, Wang W, Wang Z and Wang X: Synergistic inhibitory effects of deferasirox in combination with decitabine on leukemia cell lines SKM-1, THP-1, and K-562. Oncotarget 8: 36517-36530, 2017.

3. Rose C, Brechignac S, Vassilief D, Pascal L, Stamatoullas A, Guerci A, Larbaa D, Dreyfus F, Beyne-Rauzy O, Chaury MP, et al; GFM (Groupe Francophone des Myélodysplasies): Does iron chelation therapy improve survival in regularly transfused lower risk MDS patients? A multicenter study by the GFM (Groupe Francophone des Myélodysplasies). Leuk Res 34: 864-870, 2010.

4. Schanz J, Tüchler H, Solé F, Mallo M, Luño E, Cervera J, Granada I, Hildebrandt B, Slovak ML, Ohyashiki K, et al: New comprehensive cytogenetic scoring system for primary myelodysplastic syndromes (MDS) and oligoblastic acute myeloid leukemia after MDS derived from an international database merge. J Clin Oncol 30: 820-829, 2012.

5. Ahmad A and Iqbal MA: Significance of genome-wide analysis of copy number alterations and UPD in myelodysplastic syndromes using combined CGH - SNP arrays. Curr Med Chem 19: 3739-3747, 2012.

6. Pfeifer D, Pantic M, Skatulla I, Rawluk J, Kreutz C, Martens UM, Fisch P, Timmer J and Veelken H: Genome-wide analysis of DNA copy number changes and LOH in CLL using high-density SNP arrays. Blood 109: 1202-1210, 2007.

7. Wang L, Fidler C, Nadig N, Giagounidis A, Della Porta MG, Malcovati L, Killick S, Gattermann N, Aul C, Boultwood J, et al: Genome-wide analysis of copy number changes and loss of heterozygosity in myelodysplastic syndrome with del $(5 q)$ using high-density single nucleotide polymorphism arrays. Haematologica 93: 994-1000, 2008.

8. Bis DM, Schüle R, Reichbauer J, Synofzik M, Rattay TW, Soehn A, de Jonghe P, Schöls L and Züchner S: Uniparental disomy determined by whole-exome sequencing in a spectrum of rare motoneuron diseases and ataxias. Mol Genet Genomic Med 5: 280-286, 2017.

9. Steinbach D, Schramm A, Eggert A, Onda M, Dawczynski K, Rump A, Pastan I, Wittig S, Pfaffendorf N, Voigt A, et al: Identification of a set of seven genes for the monitoring of minimal residual disease in pediatric acute myeloid leukemia. Clin Cancer Res 12: 2434-2441, 2006.

10. Steinbach D, Bader P, Willasch A, Bartholomae S, Debatin KM, Zimmermann M, Creutzig U, Reinhardt D and Gruhn B: Prospective validation of a new method of monitoring minimal residual disease in childhood acute myelogenous leukemia. Clin Cancer Res 21: 1353-1359, 2015.

11. Neilson LI, Schneider PA, Van Deerlin PG, Kiriakidou M, Driscoll DA, Pellegrini MC, Millinder S, Yamamoto KK, French CK and Strauss JF III: cDNA cloning and characterization of a human sperm antigen (SPAG6) with homology to the product of the Chlamydomonas PF16 locus. Genomics 60: 272-280, 1999.

12. Zhang Z, Jones BH, Tang W, Moss SB, Wei Z, Ho C, Pollack M, Horowitz E, Bennett J, Baker ME and Strauss JF 3rd: Dissecting the axoneme interactome: The mammalian orthologue of Chlamydomonas PF6 interacts with sperm-associated antigen 6, the mammalian orthologue of Chlamydomonas PF16. Mol Cell Protezom 4: 914-923, 2005.

13. Sapiro R, Kostetskii I, Olds-Clarke P, Gerton GL, Radice GL and Strauss JF III: Male infertility, impaired sperm motility, and hydrocephalus in mice deficient in sperm-associated antigen 6. Mol Cell Biol 22: 6298-6305, 2002.

14. Lonergan KM, Chari R, Deleeuw RJ, Shadeo A, Chi B, Tsao MS, Jones S, Marra M, Ling V, Ng R, et al: Identification of novel lung genes in bronchial epithelium by serial analysis of gene expression. Am J Respir Cell Mol Biol 35: 651-661, 2006.

15. Mulaw MA, Krause A, Deshpande AJ, Krause LF, Rouhi A, La Starza R, Borkhardt A, Buske C, Mecucci C, Ludwig WD, et al: CALM/AF10-positive leukemias show upregulation of genes involved in chromatin assembly and DNA repair processes and of genes adjacent to the breakpoint at 10p12. Leukemia 26: 1012-1019, 2012 
16. Silina K, Zayakin P, Kalnina Z, Ivanova L, Meistere I Endżelinš E, Ábols A, Stengrévics A, Leja M, Ducena K, et al: Sperm-associated antigens as targets for cancer immunotherapy: Expression pattern and humoral immune response in cancer patients. J Immunother 34: 28-44, 2011

17. Nakagawa T, Matozaki S, Murayama T, Nishimura R, Tsutsumi M, Kawaguchi R, Yokoyama Y, Hikiji K, Isobe T and Chihara K.: Establishment of a leultaemic cell line from a patient with acquisition of chromosomal abnormalities during disease progression in myelodysplastic syndrome. Br J Haematol 85: 469-476, 1993.

18. Yang B, Wang L, Luo X, Chen L, Yang Z and Liu L: SPAG6 silencing inhibits the growth of the malignant myeloid cell lines SKM-1 and K562 via activating p53 and caspase activationdependent apoptosis. Int J Oncol 46: 649-656, 2015.

19. Li X, Yang B, Wang L, Chen L, Luo X and Liu L: SPAG6 regulates cell apoptosis through the TRAIL signal pathway in myelodysplastic syndromes. Oncol Rep 37: 2839-2846, 2017.

20. Di Cristofano A and Pandolfi PP: The multiple roles of PTEN in tumor suppression. Cell 100: 387-390, 2000.

21. Stambolic V, Suzuki A, de la Pompa JL, Brothers GM, Mirtsos C, Sasaki T, Ruland J, Penninger JM, Siderovski DP and Mak TW: Negative regulation of $\mathrm{PKB} / \mathrm{Akt}$-dependent cell survival by the tumor suppressor PTEN. Cell 95: 29-39, 1998.

22. Zhao L, Shan Y, Liu B, Li Y and Jia L: Functional screen analysis reveals miR-3142 as central regulator in chemoresistance and proliferation through activation of the PTEN-AKT pathway in CML. Cell Death Dis 8: e2830, 2017.

23. Scherr M and Eder M; M SMaE: Gene transfer into hematopoietic stem cells using lentiviral vectors. Curr Gene Ther 2 $45-55,2002$

24. Livak KJ and Schmittgen TD: Analysis of relative gene expression data using real-time quantitative PCR and the 2(-Delta Delta C(T)) Method. Methods 25: 402-408, 2001

25. Storti P, Donofrio G, Colla S, Airoldi I, Bolzoni M, Agnelli L, Abeltino M, Todoerti K, Lazzaretti M, Mancini C, et al: HOXB7 expression by myeloma cells regulates their pro-angiogenic properties in multiple myeloma patients. Leukemia 25: 527-537, 2011.

26. Song MS, Salmena L and Pandolfi PP: The functions and regulation of the PTEN tumour suppressor. Nat Rev Mol Cell Biol 13 283-296, 2012

27. Yang JY and Widmann C: The RasGAP N-terminal fragment generated by caspase cleavage protects cells in a Ras/PI3K/ Akt-dependent manner that does not rely on NFkappa B activation. J Biol Chem 277: 14641-14646, 2002.

28. Rhee I, Bachman KE, Park BH, Jair KW, Yen RW, Schuebel KE Cui H, Feinberg AP, Lengauer C, Kinzler KW, et al: DNMT1 and DNMT3b cooperate to silence genes in human cancer cells. Nature 416: 552-556, 2002 .

29. Tsoplou P, Kouraklis-Symeonidis A, Thanopoulou E, Zikos P, Orphanos V and Zoumbos NC: Apoptosis in patients with myelodysplastic syndromes: Differential involvement of marrow cells in 'good' versus 'poor' prognosis patients and correlation with apoptosis-related genes. Leukemia 13: 1554-1563, 1999.
30. Parker JE and Mufti GJ: Excessive apoptosis in low risk myelodysplastic syndromes (MDS). Leuk Lymphoma 40: 1-24, 2000.

31. Kerbauy DB and Deeg HJ: Apoptosis and antiapoptotic mechanisms in the progression of myelodysplastic syndrome. Exp Hematol 35: 1739-1746, 2007.

32. Rodriguez M, Siwko S, Zeng L, Li J, Yi Z and Liu M: Prostatespecific G-protein-coupled receptor collaborates with loss of PTEN to promote prostate cancer progression. Oncogene 35: 1153-1162, 2016.

33. Schöndorf T, Göhring UJ, Roth G, Middel I, Becker M, Moser N, Valter MM and Hoopmann M: Time to progression is dependent on the expression of the tumour suppressor PTEN in ovarian cancer patients. Eur J Clin Invest 33: 256-260, 2003.

34. Tsutsui S, Inoue H, Yasuda K, Suzuki K, Higashi H, Era S and Mori M: Reduced expression of PTEN protein and its prognostic implications in invasive ductal carcinoma of the breast. Oncology 68: 398-404, 2005.

35. Nyåkern M, Tazzari PL, Finelli C, Bosi C, Follo MY, Grafone T, Piccaluga PP, Martinelli G, Cocco L and Martelli AM: Frequent elevation of Akt kinase phosphorylation in blood marrow and peripheral blood mononuclear cells from high-risk myelodysplastic syndrome patients. Leukemia 20: 230-238, 2006.

36. Igney FH and Krammer PH: Death and anti-death: Tumour resistance to apoptosis. Nat Rev Cancer 2: 277-288, 2002.

37. Kim AH, Khursigara G, Sun X, Franke TF and Chao MV: Akt phosphorylates and negatively regulates apoptosis signalregulating kinase 1. Mol Cell Biol 21: 893-901, 2001.

38. Hu X, Yan R, Cheng X, Song L, Zhang W, Li K and Zhao S: The function of sperm-associated antigen 6 in neuronal proliferation and differentiation. J Mol Histol 47: 531-540, 2016.

39. Lee DW, Futami M, Carroll M, Feng Y, Wang Z, Fernandez M, Whichard Z, Chen Y, Kornblau S, Shpall EJ, et al: Loss of SHIP-1 protein expression in high-risk myelodysplastic syndromes is associated with miR-210 and miR-155. Oncogene 31: 4085-4094, 2012.

40. Shu Y, Zhou X, Qi X, Liu S, Li K, Tan J, Liu Z, Yu J, Zhang P and Zou L: $\beta$-Arrestin1 promotes the self-renewal of the leukemiainitiating cell-enriched subpopulation in B-lineage acute lymphoblastic leukemia related to DNMT1 activity. Cancer Lett 357: 170-178, 2015.

41. Altenberger C, Heller G, Ziegler B, Tomasich E, Marhold M, Topakian T, Müllauer L, Heffeter P, Lang G, End-Pfützenreuter A, et al: SPAG6 and L1TD1 are transcriptionally regulated by DNA methylation in non-small cell lung cancers. Mol Cancer 16: 1, 2017.

42. Fuks F, Burgers WA, Brehm A,Hughes-Davies L and Kouzarides T: DNA methyltransferase Dnmt1 associates with histone deacetylase activity. Nat Genet 24: 88-91, 2000.

43. Hermann A, Gowher $\mathrm{H}$ and Jeltsch A: Biochemistry and biology of mammalian DNA methyltransferases. Cell Mol Life Sci 61 : 2571-2587, 2004. 